\title{
Transitions of Care in the Perioperative Period
}

\author{
Andrew M. Becker ${ }^{1} \cdot$ Meghan Lane-Fall ${ }^{1}$
}

Published online: 23 October 2017

(C) Springer Science+Business Media, LLC 2017

\begin{abstract}
Purpose of Review The purpose of this article is to summarize the evolving literature concerning perioperative handoffs for surgical patients.

Recent Findings Perioperative transitions of care are high-risk events with the opportunity for patient harm. Recognition of this risk has led to increased scrutiny of handoffs occurring throughout the perioperative care continuum. Though limited, previous literature has focused on the use of checklists as a strategy to increase handoff reliability. More recent literature suggests that broader, process-driven interventions may be more effective in addressing the multiple factors that contribute to the success of patient handoffs. Further, recent studies have begun to address the sustainability of such interventions. Summary Handoffs are complex, error-prone patient care events. Handoff process standardization (including, but not solely consisting of checklist use) is a promising, evidencebased strategy that has been shown to improve communication and mitigate harm to patients receiving care in perioperative settings.
\end{abstract}

Keywords Surgery · Anesthesia · Perioperative · Transition · Handoff · Communication · Implementation · Quality improvement $\cdot$ Education $\cdot$ Intensive care unit

This article is part of the Topical Collection on Patient Safety in Anesthesia

Meghan Lane-Fall

Meghan.Lane-Fall@uphs.upenn.edu

1 Department of Anesthesiology and Critical Care, Perelman School of Medicine, University of Pennsylvania, 309 Blockley Hall, 423 Guardian Drive, Philadelphia, PA 19104-4865, USA

\section{Introduction}

Communication in the hospital setting continues to be a focal point of patient safety efforts given robust evidence associating healthcare communication defects with patient harm. In the perioperative arena, this focus on communication has been reflected in an increasing number of studies examining communication across the perioperative care continuum, with a particular focus on handoffs within and between perioperative care settings. In this review, we describe the recent evidence on handoffs as patients transition across perioperative care settings, highlighting those studies that demonstrate the association between handoffs and patient outcomes as well as studies reporting interventions to improve handoff reliability.

Since the 2007 Joint Commission National Patient Safety Goal [1] and the more recent Sentinel Event Alert on handoffs [2], emphasis has been placed on understanding the implementation, effectiveness, and outcomes of patient care handoffs and handoff interventions. Further, the Accreditation Council on Graduate Medical Education made handoff curricula addressing transitions of care a mandatory part of residency training in its 2011 Common Program Requirements [3,4]. This educational mandate is meant to ensure that residency training programs are responsive to the evidence showing that handoffs directly affect patient outcomes [5].

Patient care handoffs share similar features with exchanges of information in high-consequence, high-reliability organizations. Patterson et al. identified 21 different strategies to support information exchange that were employed by multiple high-risk industries including NASA and nuclear power plants [6]. Scripted practices for communication in these settings include the face-to-face transfer of information, the creation of an interruption-free space, and a process of giving the rationale and context for expected changes to the plan moving 
forward. Leveraging lessons from another high-risk industry, Catchpole et al. collaborated with Formula-1 racing pit-stop crews to build a handoff tool for the pediatric surgery-to-ICU transition of care that employed many of these features [7].

Despite signals that handoff process standardization might be an effective strategy, Keebler et al. showed in an extensive meta-analysis that there is widespread variation in both the methods of handoff implementation and the interventions themselves $[8 \bullet]$. However, they were able to show that handoff standardization improved all studied outcome measures, including handoff information (amount of patient information transferred), patient outcomes (adverse events and complications), process outcomes (use of handoff, time spent, and presence of interruptions), and provider and organizational outcomes (satisfaction and culture). Keebler and colleagues' meta-analysis was not limited to surgical settings, but their findings have important implications for perioperative handoffs.

As we consider perioperative handoffs, we note that nontechnical communication skills like those needed for effective handoffs are integral to surgical care [9]. Considering this need, there is a surprising paucity of literature supporting particular strategies to facilitate high-quality perioperative transitions of care. However, the past 10 years have seen a significant uptick in the number of studies reporting interventions to improve perioperative handoffs.

\section{Location and Direction Matters}

Handoffs, which we define as transfers of patient care information, responsibility, and accountability [10], are an integral feature of surgical care. For example, surgical inpatient care may include handoffs from pre-admission settings to the preoperative holding area, then to the operating room (OR), then to either the post-anesthesia care unit (PACU) or intensive care unit (ICU), and finally to discharge (Fig. 1). There may be other transitions as well, including those from the emergency department to the OR. Beyond transitions of environment, handoffs occur within locations, such as the intraoperative handoffs between anesthesia staff, or nurse shift change handoffs within the PACU or ICU. Here, we focus on "transitions in care," a particular type of handoff that occurs as patients move from setting to setting [4].

\section{OR-to-PACU Handoffs}

There are multiple published studies demonstrating deficient information exchange at the time of OR-to-PACU handoffs and subsequent improvement in information exchange with standardization. Petrovic et al. successfully transplanted a previously reported OR-to-ICU handoff standardized process to the OR-to-PACU setting [11]. In their study, they reference the challenges required to translate a previous intervention into a similar, yet new setting. The study team added specialtyspecific checklists for the anesthesiologist and surgeon completing the handoff. They showed fewer information omissions and technical errors, as well as increased PACU nurse satisfaction after implementation. Other studies published in the past 5 years show similar results, favoring structured ORto-PACU handoffs [12-14].

\section{OR-to-ICU Handoffs}

There are more than 20 published studies addressing the transition of patients from the OR to the ICU, with at least 10 published in the last 5 years [15-25]. This particular transition is interesting for multiple reasons. First, patients generally move longer distances during an OR-to-ICU transport than OR-to-PACU, which increases the length of time during which transport-related mishaps can occur. Second, patients are more often unstable or at high risk of deterioration, which increases the stakes of the handoff. Third, ICU providers are not solely focused on perioperative care (i.e., they have competing patient care priorities), and the OR-to-ICU handoff can represent a disruption in workflow. To our knowledge, all published studies of OR-to-ICU handoffs have reported interventions leading to a standardized handoff procedure, and they have all shown improvements in information exchange. Granted, there may be publication bias favoring studies with positive results, but the existing body of evidence does support structured OR-to-ICU handoffs.

\section{ICU-to-OR Handoffs}

A patient's course in the hospital is not always linear. For example, the patient may progress off the floor to the ICU days after an operative procedure, or may cycle from the OR to the ICU, then back to the OR for revisions or emergency procedures. Evans et al. described what they called the "reverse handoff," from the ICU to the OR [26]. In their article, they suggest this handoff may be as problematic as handoffs that occur more frequently, considering patients moving towards the OR from the ICU are often unstable with emergent, unplanned, off-hours surgical procedures. Caruso et al. studied the ICU-to-OR handoff, describing the implementation of a multidisciplinary handoff practice using similar principles to handoffs studied in other settings, including process standardization and ensuring face-to-face communication. The implementation of their handoff procedure was found to increase the percentage of ICU-to-OR handoffs occurring, increase patient preparedness, and did not postpone or delay the timing of surgery [27].

Clearly, patients experience multiple perioperative transitions of care as they receive surgical care. At these transitions, effective communication is paramount, no matter the location 
Fig. 1 Schematic depicting transitions of care for patients undergoing inpatient surgery. Shading indicates whether there is published literature addressing handoffs within a given setting (shaded boxes) or between settings (shaded arrows). The width of each arrow indicates relative volume of patient flow (i.e., thinner arrows represent less patient flow between areas as compared to transitions with wider arrows)

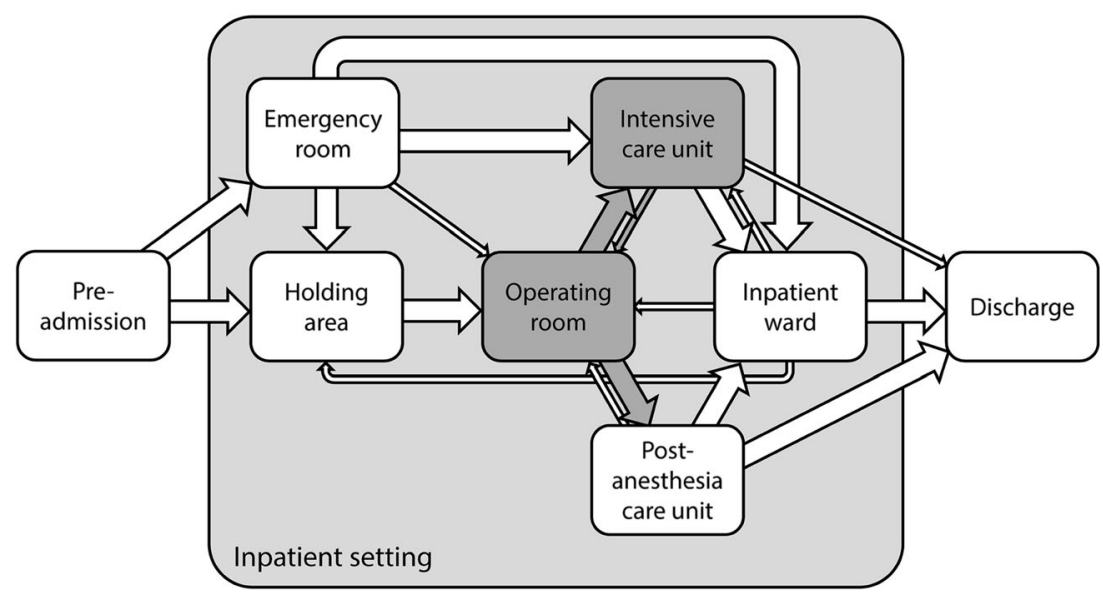

or direction of transfer. The majority of the perioperative handoff literature addresses OR-to-PACU and OR-to-ICU handoffs, and so further research is needed to understand and improve handoffs in other perioperative locations. However, the handoff literature in surgical and non-surgical settings alike is converging on standardization as a strategy to improve handoff reliability. For this reason, it is important to understand efforts to standardize handoffs.

\section{Standardization with (Just) a Checklist}

Early efforts to standardize handoffs focused primarily on the information exchange function of handoffs and implemented checklists to improve the exchange of information. Handoff standardization with checklists involves the creation of paper or electronic lists of information deemed important to be included in the handoff.

Of note, the first randomized control trial of a handoff intervention-a checklist - was recently reported by Salzwedel et al., who created an information checklist for the OR-to-ICU handoff. In their study, attending anesthesiologists noted information that "must be handed over" and which information "should be handed over" for each patient they cared for while in the operating room [28]. The resident anesthesiologist or advanced practitioner completing the handoff received a closed envelope on arrival to the ICU with or without the checklist, which was randomized by patient case. The handoff was voice recorded. This information was then compared against the attending anesthesiologist's list of "must be handed over" or "should be handed over" completed intraoperatively. The study reports that the intervention group increased their percentage of "must" and "should" items handed-over compared to controls. However, it must be noted that there is high likelihood of contamination with RCTs in this setting, considering randomized anesthesiology residents most likely had participated and used the checklist for other patients who had been randomized to be included, which may have affected their subsequent behavior. There is also the possibility that the presence of a checklist in the envelope affected behavior irrespective of the contents of the checklist.

Other recent studies of checklist standardization have shown positive results as well. This includes a study by Funk et al. that showed the introduction of a PACU handoff checklist improved the number of items discussed within the introduction, background, assessment, and clinical recommendations portions of the handoff [13]. Notably, standardization with checklists may also improve the process of the handoff itself. Bavare et al. show that the implementation of a pocket-sized verbal checklist showed improvements in the communication process, including the identification of the patient, relevant history noted, as well as a decrease in interruptions during the sign-out [29].

The ideal components of a handoff checklist are not yet known and probably vary based on the specific perioperative setting. However, Keebler et al. suggest that that longer checklists (> 12 items) are associated with increases in the amount of information transferred [8•].

As the literature evolves, standardization efforts seem to include more than just checklists, as checklists address only the information transfer function of handoffs. We are starting to see more studies that are interested in standardizing the entire handoff process, including not just information, but also a "choreography"-who should participate and when, and setting expectations about the nature of these interactions.

\section{Standardization with a Process}

Early efforts to standardize the handoff began with straightforward checklists delineating key patient-related information for exchange. This approach often yielded improved information exchange for items addressed by the checklists, but did not address the non-information-related aspects of handoffs [10], i.e., they did not take the broader view of handoffs as a process requiring co-construction of a shared mental model 
about the patient that ideally includes shared creation of a patient care plan. A different approach taken by more recent studies is the development and implementation of processdriven protocols.

Handoff protocols address clinician roles, movement, communication, and expectations of the multidisciplinary team involved in surgical transitions of care. Effective handoff protocols facilitate communication between multiple providers, improve efficiency of movement, and decrease information redundancy. Checklists are often used in handoff protocols, but protocols are more expansive than checklists alone. LaneFall et al. provide an example of common features of OR-toICU handoff protocols in their 2014 paper [30].

Literature suggests some important approaches to developing and implementing standardized handoff processes. Segall et al. took a multidisciplinary "human-centered design" approach, bringing key stakeholders together to redesign ORto-ICU handoffs. They found that use of their process increased team behavior scores (percent of listed communication behaviors witnessed). They suggested that key aspects of improving handoffs include clarifying roles of providers and building checklist-like information forms [18•].

Using a different approach to facilitate communication between OR and ICU teams, Breuer et al. implemented a threepart standardized process by which communication occurs between these two teams both intra- and postoperatively [31]. First, they instituted standardized intraoperative calls from the OR to the pediatric ICU. Second, they implemented a postoperative "time-out" approach where all required provider parties needed to be present to commence the handoff. Third, a standardized checklist of information was built for the anesthesiologist's verbal handoff. This study showed an improved transfer of information with decreased information omissions and increased frequency of discussion of post-op treatment plans. Notably, their study also showed early improvement in patient outcomes. This included a decreased number of patients receiving antibiotic administration delays, decreased patients requiring additional fluid boluses or vasopressors, improved pain scores, and decreased number of patients requiring escalating respiratory support.

Other literature has recently been published on the standardization of the handoff process across different units. Halvorson et al. implemented a workflow standardization process that they applied to improve the variation noted across their hospital's four ICU units (medical, neuro, cardiac, and surgical). Their process involved an organized approach by the unit charge nurse, bed management, environmental services, ICU nurse, and physician providers [32]. After implementation, they reported improved information communication, unchanged time to transport, and $100 \%$ adherence to the process at 12 months post implementation.

Other studies institute more targeted process improvements aimed at one aspect of the handoff process. For example,
Moon et al. showed that a prescribed pre-transport phone call from anesthesia provider to ICU nurse improved physician provider and nurse provider satisfaction with the hand-over process [19]. In addition, this process improvement was "bundled" with a mnemonic that was created and placed strategically to help guide the inclusion of key information during the standardized phone call. Finally, the postoperative anesthesia note was standardized so pertinent information was available in the immediate postoperative setting.

There have similarly been studies that standardize the expected roles for each member of the multidisciplinary provider team during patient transport and their location within the room during the handoff. Vergales et al. created a standardized process with which the PICU nurse was physically involved in the OR-to-PICU patient transfer and standardized roles for each provider in the room once transport to PICU was complete [22]. A written information "checklist" was built into the process to assure the appropriate transfer of information. Their study showed strong adherence to the new transport process as well as provider survey that suggested the process improved patient care. In a similar fashion, Kaufman et al. implemented a process standardization for the cardiovascular OR to the cardiovascular ICU that defined key roles within the multidisciplinary team as well as these providers' placement around the bedside [33]. Their study linked these process improvements to patient outcomes. This included a decrease in unplanned extubations and median ventilator time after the handoff process implementation as compared to the period prior to implementation.

Lastly, there are components of the handoff that may prove to be unexpected yet important aspects of the process. Moving beyond the patient, Stutzman et al. shown that the family of the patient may also be an area of the handoff to be considered [34]. They discovered qualitative themes of the handoff by interviewing a select set of family members for patient transitions from OR to ICU. Themes that the interviewees deemed helpful were direct communication by the provider team to the family, witnessing nurse-to-nurse discussion about the patient, physicians interacting with a "confident" demeanor, and physicians who were perceived as "accessible" in the perioperative period.

\section{Sustainability}

As new handoff protocols become engrained within their respective institutions, follow-up research should evaluate their sustainability, longevity, and effectiveness over time. Of the above cited studies, only two address the sustainability of their interventions. Breuer et al. showed sustained improvement in communication measures, but only sustained improvement for a single patient outcome measure (decreased number of patients with delayed doses of antibiotics) at 1 year [31]. 
Kaufman et al. similarly tracked outcome measures beyond the immediate post-implementation period. Their study showed that their process standardization intervention was associated with improvements in unplanned extubations and median ventilator time at 2 years post implementation [33].

Quality improvement strategies may be applied to the handoff interventions to improve their sustainability. Riley et al. suggest that creating a unit-based (and even institutional) culture of safety around a handoff implementation may yield dividends in sustainability. In their follow-up study, they report continually improving information handoff metrics at 4 years post implementation. Further, they suggest promoting a "shared mental model," where multiple providers involved in the handoff process share similar goals and reverence for the importance of the intervention [35••], which may help maintain process adherence over time.

Similarly, Chenault et al. published a follow-up study on information checklist initially implemented in 2011. They show that 5 years after implementation, the number of information omissions is lower than both pre-intervention and the post-intervention phases of the handoff implementation [20]. Further, they show that the sustainability phase has a decreased time length of the handoff. While there was no difference in technical errors between the sustainability phase and the previous phases, this study shows that embedding a change in the workflow culture of a unit can have positive impacts on the change even years after initial implementation.

\section{Conclusion}

The handoff is an important process for the safe transition of care for patients in the perioperative setting. Research in highrisk industries reminds us that effective, reliable communication is a hallmark of highly reliable organizations $[6,7]$. Handoffs in the healthcare setting are unavoidable for surgical patients, and communication breakdowns can readily translate into avoidable harm. Fortunately, an increasing number of studies demonstrate decreases in avoidable harm when handoff standardization is employed [5, 31, 33].

Transitions of care for the surgical patient are numerous and present their own setting-specific challenges. Most published studies have focused on the OR-to-PACU and OR-toICU transitions. Yet, recent studies have begun to shed light on other equally as challenging transitions of care including the ICU-to-OR handoff [27].

Early efforts to standardize the handoff began with straightforward checklists delineating key patient-related information for exchange $[13,28,29]$. This approach often yielded improved information exchange for items addressed by the checklists, but did not address the non-information-related aspects of handoffs [10], i.e., they did not take the broader view of handoffs as a process. More recent studies have standardized the entire handoff process by addressing the roles, movements, communication, and expectations of the multi-disciplinary team involved in surgical transitions of care. Continued handoff standardization implementation must appreciate the handoff as a broader process of care beyond just the communication of key facts.

As with any quality improvement efforts, the sustainability of these implementations must be continually evaluated with sensitive and specific metrics. Follow-up studies should be conducted both to advance our understanding of the handoff and to critically remind us that long-term progress often requires sustained effort and appraisal. As continued research gives insight to handoff design, implementation, and sustainability, continued system wide education and focus will be crucial. Considering the risks of communication error, the successful study of handoffs in the perioperative setting will continue to be of the utmost importance for high-quality surgical care.

\section{Compliance with Ethical Standards}

Conflict of Interest Andrew M. Becker and Meghan Lane-Fall declare they have no conflict of interest.

Human and Animal Rights and Informed Consent This article does not contain any studies with human or animal subjects performed by any of the authors.

\section{References}

Papers of particular interest, published recently, have been highlighted as:

- Of importance

•- Of major importance

1. The Joint Commission announces the 2009 National Patient Safety Goals and requirements. Joint Commission Perspectives. 2008;28(7):1, 11-15.

2. Joint Commission. Inadequate hand-off communication. 2017.

3. Nasca TJ, Day SH, Amis ES Jr. The new recommendations on duty hours from the ACGME task force. N Engl J Med. 2010;363(2):e3.

4. Lane-Fall MB, Brooks AK, Wilkins SA, Davis JJ, Riesenberg LA. Addressing the mandate for hand-off education: a focused review and recommendations for anesthesia resident curriculum development and evaluation. Anesthesiology. 2014;120(1):218-29.

5. Starmer AJ, Spector ND, Srivastava R, et al. Changes in medical errors after implementation of a handoff program. N Engl J Med. 2014;371(19):1803-12.

6. Patterson ES, Roth EM, Woods DD, Chow R, Gomes JO. Handoff strategies in settings with high consequences for failure: lessons for health care operations. Int J Qual Health Care. 2004;16(2):125-32.

7. Catchpole KR, De Leval MR, McEwan A, et al. Patient handover from surgery to intensive care: using formula 1 pit-stop and aviation models to improve safety and quality. Paediatr Anaesth. 2007;17(5):470-8.

8. Keebler JR, Lazzara EH, Patzer BS, et al. Meta-Analyses of the Effects of Standardized Handoff Protocols on Patient, Provider, and 
Organizational Outcomes. Human Factors. 2016;58(8):11871205. . This systematic review and meta-analysis is a useful summary of handoff intervention studies and describe standardization-related improvements in process and patient outcomes.

9. Wilson JL, Whyte RI, Gangadharan SP, Kent MS. Teamwork and communication skills in cardiothoracic surgery. Ann Thorac Surg. 2017;103(4):1049-54.

10. Patterson ES, Wears RL. Patient handoffs: standardized and reliable measurement tools remain elusive. Jt Comm J Qual Patient Saf. 2010;36(2):52-61.

11. Petrovic MA, Aboumatar H, Scholl AT, et al. The perioperative handoff protocol: evaluating impacts on handoff defects and provider satisfaction in adult perianesthesia care units. J Clin Anesth. 2015;27(2):111-9.

12. Van Der Walt JJN, Scholl AT, Joubert IA, Petrovic MA. Implementation of a postoperative handoff protocol. S Afr J Anaesth Analg. 2016;22(6):33-7.

13. Funk E, Taicher B, Thompson J, Iannello K, Morgan B, Hawks S. Structured handover in the pediatric postanesthesia care unit. J Perianesth Nurs. 2016;31(1):63-72.

14. Weinger MB, Slagle JM, Kuntz AH, et al. A multimodal intervention improves postanesthesia care unit handovers. Anesth Analg. 2015;121(4):957-71.

15. Barry ME, Hochman BR, Lane-Fall MB, et al. Leveraging Telemedicine Infrastructure to Monitor Quality of Operating Room to Intensive Care Unit Handoffs. Academic Medicine. 2017; 92(7):1035-1042.

16. Hochman BR, Barry ME, Lane-Fall MB, et al. Handoffs in the intensive care unit. Am J Med Qual. 2017;32(2):186-93.

17. Hall M, Robertson J, Merkel M, Aziz M, Hutchens M. A structured transfer of care process reduces perioperative complications in cardiac surgery patients. Anesth Analg. 2017;125(2):477-82.

18. Segall N, Bonifacio AS, Barbeito A, et al. Operating room-to-ICU patient handovers: a multidisciplinary human-centered design approach. Jt Comm J Qual Patient Saf. 2016;42(9):400-14. This paper demonstrates the use of human factors principles in designing an OR to ICU handoff process that complements clinician workflow

19. Moon TS, Gonzales MX, Woods AP, Fox PE. Improving the quality of the operating room to intensive care unit handover at an urban teaching hospital through a bundled intervention. J Clin Anesth. 2016;31:5-12.

20. Chenault K, Moga MA, Shin M, et al. Sustainability of protocolized handover of pediatric cardiac surgery patients to the intensive care unit. Paediatr Anaesth. 2016;26(5):488-94.

21. McElroy LM, Collins KM, Koller FL, et al. Operating room to intensive care unit handoffs and the risks of patient harm. Surgery (United States). 2015;158(3):588-94.

22. Vergales J, Addison N, Vendittelli A, et al. Face-to-face handoff: improving transfer to the pediatric intensive care unit after cardiac surgery. Am J Med Qual. 2015;30(2):119-25.
23. Dixon JL, Stagg HW, Wehbe-Janek H, Jo C, Culp WC Jr, Shake JG. A standard handoff improves cardiac surgical patient transfer: operating room to intensive care unit. J Healthc Qual. 2015;37(1):2232.

24. McElroy LM, Macapagal KR, Collins KM, et al. Clinician perceptions of operating room to intensive care unit handoffs and implications for patient safety: A qualitative study. American Journal of Surgery. 2015; 210(4): 629-35.

25. Lane-Fall M, Collard M, Gutsche J, et al. Mixed-methods evaluation and standardization of OR to ICU handoffs in a mixed surgical population. American Society of Anesthesiologists Annual Meeting; October, 2015; San Diego, CA.

26. Evans AS, Yee MS, Hogue CW. Often overlooked problems with handoffs: from the intensive care unit to the operating room. Anesth Analg. 2014;118(3):687-9.

27. Caruso TJ, Marquez JLS, Gipp MS, Kelleher SP, Sharek PJ. Standardized ICU to OR handoff increases communication without delaying surgery. Int J Health Care Qual Assur. 2017;30(4):304-11.

28. Salzwedel C, Bartz HJ, Kuhnelt I, et al. The effect of a checklist on the quality of post-anaesthesia patient handover: a randomized controlled trial. Int J Qual Health Care. 2013;25(2):176-81.

29. Bavare AC, Shah PK, Roy KM, Williams EA, Lloyd LE, McPherson ML. Implementation of a Standard Verbal Sign-Out Template Improves Sign-Out Process in a Pediatric Intensive Care Unit. Journal for Healthcare Quality. 2013; 37(5):267-276.

30. Lane-Fall MB, Beidas RS, Pascual JL, et al. Handoffs and transitions in critical care (HATRICC): protocol for a mixed methods study of operating room to intensive care unit handoffs. BMC Surg. 2014;14:96.

31. Breuer RK, Taicher B, Turner DA, Cheifetz IM, Rehder KJ. Standardizing postoperative PICU handovers improves handover metrics and patient outcomes. Pediatr Crit Care Med. 2015;16(3): 256-63.

32. Halvorson S, Wheeler B, Willis M, et al. A multidisciplinary initiative to standardize intensive care to acute care transitions. Int J Qual Health Care. 2016;28(5):615-25.

33. Kaufman J, Twite M, Barrett C, et al. A Handoff Protocol from the Cardiovascular Operating Room to Cardiac ICU Is Associated with Improvements in Care Beyond the Immediate Postoperative Period. Joint Commission Journal on Quality and Patient Safety. 2013;39(7):306-311.

34. Stutzman SE, Olson DM, Greilich PE, Abdulkadir K, Rubin MA. The patient and family perioperative experience during transfer of care: a qualitative inquiry. AORN J. 2017;105(2):193-202.

35.• Riley CM, Merritt AD, Mize JM, Schuette JJ, Berger JT. Assuring Sustainable Gains in Interdisciplinary Performance Improvement: Creating a Shared Mental Model During Operating Room to Cardiac ICU Handoff. Pediatric Critical Care Medicine. 2017; 18(9):863-868. This study is one of the first to address sustainability of an OR to ICU handoff standardization intervention. 